\title{
GENERALIZED POWER Allocation (GPA) SCHEME FOR NON-ORTHOGONAL MULTIPLE ACCESS (NOMA) BASED WIRELESS COMMUNICATION SYSTEM
}

\author{
Tofail Ahmed ${ }^{1}$, Rubaiyat Yasmin², Halida Homyara² and M.A.F.M Rashidul \\ Hasan $^{2}$ \\ ${ }^{1}$ Department of Information and Communication Engineering, Bangladesh Army \\ University of Engineering and Technology, Quadirabad Cantonment, Natore, Bangladesh \\ ${ }^{2}$ Department of Information and Communication Engineering, University of Rajshahi, \\ Rajshahi, Bangladesh
}

\begin{abstract}
This paper presents a Generalized Power Allocation (GPA) scheme for different users in Non-Orthogonal Multiple Access (NOMA) based wireless communication system. The power allocation to the users becomes complex with the increased number of users. There are some conventional schemes for power allocation in NOMA but they have to optimize some parameters arbitrarily. In this paper, a simple but effective power allocation scheme has been formulated and tested by simulations. The proposed GPA scheme does not need any parameter adjustment. Theoretical power distribution to different users of NOMA has been calculated using the proposed GPA technique. The calculated powers of individual users with the proposed scheme are different and more distributed than the arbitrary power allocation scheme which satisfies the basic condition of NOMA. The total of calculated powers with GPA scheme shows only 01\% variation with the arbitrary power allocation scheme which shows the consistency of GPA scheme with other schemes. The performance of NOMA based wireless communication system with GPA scheme has been simulated under various conditions using Matlab. The simulated BER performance for NOMA based wireless communication system using different modulation techniques show similar results with other conventional schemes which validates the formulation of GPA scheme.
\end{abstract}

\section{KEYWORDS}

BER, power allocation, OFDMA, NOMA, FTPA, GPA, fairness index.

\section{INTRODUCTION}

The rapid growth of innovations in information and communication technology area has greatly emphasized the wireless communication experience of current generation. In future, new generation are expected to be surrounded by smart objects in smart homes, offices, streets, and cities in the smart world [1]. Researchers and network service providers face many challenges due to the exponential growth of data traffic and connected devices. In this context, we need to improve network performance in terms of throughput and capacity, or number of users. However, the radio resources, the wireless spectrum and transmit power are limited and current wireless communication technologies are struggling to accommodate such increase in the traffic demand within the available resources. The technologies and new ideas are needed to improve the capacity and to provide high data rate. At multi-carrier wireless communication systems, Orthogonal Frequency Division Multiplexing (OFDM) has been widely adopted based on its 
advantage of transforming a frequency selective fading channel into a number of narrowband flat fading sub channels. Wireless multiple access technique can be realized in two ways: Orthogonal Frequency Division Multiple Access (OFDMA) [2] and Non-Orthogonal Multiple Access (NOMA) [3]. In view of increasing the spectral efficiency of the next generation networks and reduction of inter cell interference levels, NOMA technique seems to be promising [4]. All the users in NOMA use the same time and frequency resources which results in improved spectral efficiency but power is different for all users. Hence NOMA is a power domain multiplexing scheme [5]. However there are many challenges in NOMA to transmit the signal in efficient ways. One of them is to handle the power allocation of different users in efficient ways; hence we focused on power allocation technique for NOMA. Because in NOMA system, the frequency spectrum is not divided likes OFDMA. Rather the frequency is same but the power is different for different users in NOMA based wireless communication system. The application of NOMA for $5 \mathrm{G}$ communication is being studied [6-8] and explored.

\section{RELATED WORKS}

The selection of suitable power allocation technique is crucial to make NOMA more effective. Paper [4, 5] showed the performance of NOMA by dividing the power to different users arbitrarily, $20 \%$ of total power is allocated to one user and $80 \%$ is allocated to other user without any mathematical modeling. In paper [9], the power for different users for NOMA have been also done arbitrarily, there is no specific formulation or model of power allocation technique. Therefore, there is no specific option of diving power as the number of user increases. On the other hand there are some specific power allocation techniques. Paper [10] is one of them where Fractional Transmit Power Allocation (FTPA) scheme has been used. In this scheme, a parameter named 'fairness index' is to be optimized arbitrarily. Paper [11] also shows the calculation of fairness index for power allocation to multiple users and need to be optimized for effective result. Therefore we focused to formulate a generalized power allocation scheme [14] for NOMA where no optimization is needed and designed for multiple users.

The rest of the paper is organized as follows. Section 3 introduces basic NOMA system design with algorithm. Section 4 discusses the conventional power allocation schemes for NOMA. In section 5, we have proposed a generalized power allocation scheme and calculated individual powers for multiple users using our proposed scheme. The results with comparative study has been shown and discussed in section 6 . The paper is concluded with our findings and future work in section 7 .

\section{NOMA SYSTEM DESIGN}

A two user case is assumed with single transmitter, and a single receiver antenna. The overall system transmission bandwidth is assumed to be $1 \mathrm{~Hz}$. The base station transmits a signal for User 1 and User 2 which is represented as $x_{i}$ with transmission power $P_{i}(\mathrm{i}=1,2)$. The sum of $P_{i}$ is restricted to be the total power $P$ at maximum. In the NOMA, $x_{1}$ and $x_{2}$ are superposition coded [13] as

$$
X=\sqrt{p_{1} x_{1}}+\sqrt{p_{2} x_{2}}
$$

The received signal at both users is represented as

$$
Y_{i}=h_{i} X+w_{i},
$$

where $h_{\mathrm{i}}$ is the complex channel coefficient between users and the base station [8]. Term $w_{i}$ denotes the receiver Gaussian noise including inter-cell interference. 


\subsection{NOMA Structure for Transmisson}

Fig. 1 shows that the NOMA uses a base station transmission scheme.

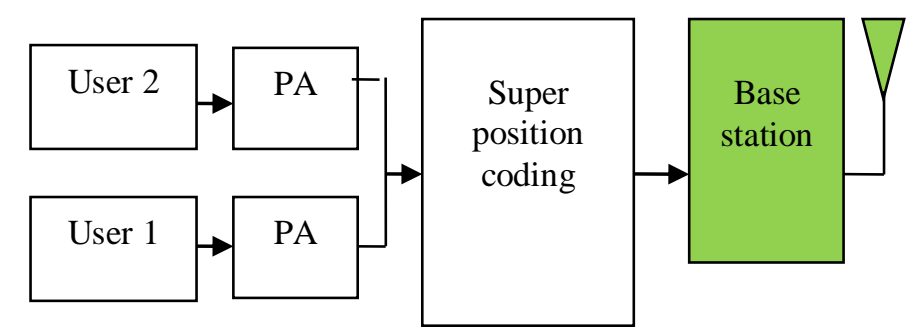

Fig. 1.Block diagram of NOMA in transmission section

Different users use the same frequency resources but are multiplexed in power domain. Considering two users User 1 and User 2 in a cell, with the power allocation (PA) technique the power of User 1 is superimposed to the power of User 2. The power of two users is different but the frequency is same. Using equation (1), the transmitted signal $\mathrm{X}$ is calculated as the total of the signal of User 1 multiplexed with $\sqrt{ } P_{1}$ and the signal of User 2 multiplexed with $\sqrt{ } P_{2}$.

\subsection{Structure for Receiption}

In transmission section of NOMA, signals emitted from the BS for each user are superimposed on top of each other. More power is allocated to user located farther from the BS. In the network, all users receive the same signal which contains the information for all users. Each user decodes the strongest signal first and it decode from the received signal. The figure of receiver section is given bellow.
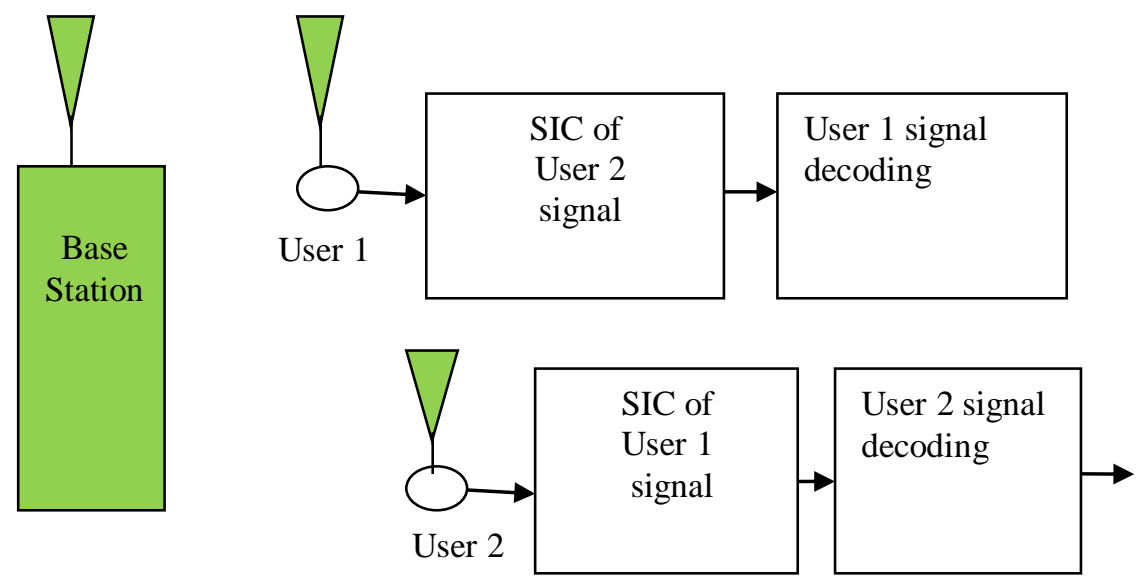

Fig. 2.Block diagram of receiver section

\subsection{Algorithm for NOMA based Appraoch}

A general algorithm of NOMA based approach is shown below:

1. Choose total power and bandwidth for NOMA based system

2. Keep the bandwidth same for every users

3. Divide the total power into individual users using proposed scheme 
4. Assign larger power for far users

5. Calculate the transmit signal using superposition coding using individual signal and individual powers

6. Transmit the signal through channel

\section{Conventional Power Allocation Schemes For Noma}

Different power allocation schemes for users are there for NOMA based wireless communication system. In this section, we discussed two popular but simple conventional techniques.

\subsection{Arbitrary Power Allocation Technique}

In paper [4, 9], the total transmit power $P$ is arbitrarily divided into users. $P_{I}$ is chosen to be $20 \%$ of total power for User 1 and, $P_{2}$ is $80 \%$ of the total power for the User2. However, it has no explicit mechanism or explanation for choosing these specific powers for users. Therefore, if the number of users are increased, the choice of power allocation will be complex.

\subsection{Fractional Transmit Power Allocation (FTPA)}

The total transmit power $P$ is fractionally divided into users based on their corresponding channel gain using fractional transmit power allocation $[10,15]$. Based on the corresponding users channel gains, the power allocation factors for two users are selected as $a_{1}$ for User 1 and $a_{2}$ for User 2 where $a_{1}<a_{2}$. Each user is loaded with power $\alpha P$ followed by superposition of all constellation points. The power allocation factor is calculated using fairness index [11]. The fairness index is optimized to be 0.7 arbitrarily. Therefore we concentrated on formulization of an effective power allocation scheme for NOMA.

\section{Proposed Generalized Power Allocation (GPa) Scheme For NOMA}

\subsection{Formulation of GPA for NOMA}

As power allocation is a crucial point for NOMA, we have proposed a simple general formula which satisfies the power allocation requirement of NOMA. The equation of the proposed Generalized Power Allocation (GPA) scheme is represented as

$$
P_{r}=\frac{n !}{r ! \times(n-r) !} \times C^{r}
$$

where $C$ is termed as Choice Factor and is calculated as follows

$$
C=p^{1 / n}-1
$$

Using the equation (3), the power of all individual users $P_{1}, P_{2}, P_{3}, \ldots \ldots, P_{\mathrm{r}}$ have been allocated where $r=1,2,3 \ldots \ldots . . n$. The equation shows that the power allocation of all users cannot be same which is the basic requirement of the NOMA system. The sum of the allocated power for individual users must satisfy the following equation.

$$
P \approx \sum_{r=1}^{n} \mathrm{P}_{\mathrm{r}}
$$




\subsection{Individual Power Calculation using Proposed GPA Scheme for NOMA}

To verify equation (3) and (5), we have calculated individual power for 6 users. We have considered total power $P$ to be 100 watt. The calculated powers for 6 users with our proposed scheme have been shown in Table 1 and Fig.3.

TABLE 1: POWER ALLOCATION FOR 6 USERS

\begin{tabular}{|c|c|c|}
\hline $\begin{array}{c}\text { Power } \\
\text { notation of } \\
\text { Users }\end{array}$ & $\begin{array}{c}\text { Calculated } \\
\text { powers(watt) }\end{array}$ & $\begin{array}{l}\text { Total } \\
\text { Power of } 6 \\
\text { Users }\end{array}$ \\
\hline$\overline{P_{1}}$ & 2 & \multirow{6}{*}{$\sum_{\mathrm{r}=1}^{6} P \mathrm{r} \approx 100$} \\
\hline$\overline{\mathrm{P}_{2}}$ & 7 & \\
\hline $\mathrm{P}_{3}$ & 12 & \\
\hline $\mathrm{P}_{4}$ & 20 & \\
\hline $\mathrm{P}_{5}$ & 27 & \\
\hline $\mathrm{P}_{6}$ & 31 & \\
\hline
\end{tabular}

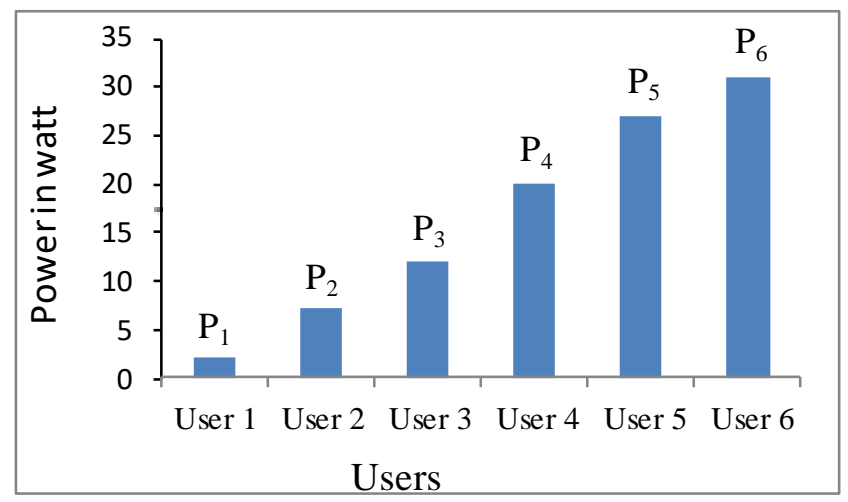

Fig. 3. Calculated power for 6 users with GPA scheme

The Table 1 and Fig. 3 show that the power of individual user is not same, they are distinguishably different with each other which is a prerequisite for NOMA power allocation. The power of users from User1 to User6 is gradually increased which is needed for near user and far user. The near user is needed small power than far user. The table also shows that the sum of individual user power is approximately equal to the total power which also satisfies the equation (5). Therefore, our formulated GPA scheme is simple and satisfies the basic prerequisites of power allocation for NOMA.

\section{RESULTS AND DiscusSiON}

We have presented simulation results using MATLAB to verify our formulized GPA scheme and compared the effectiveness of the proposed GPA schemes for NOMA with other schemes in this section. We used synthetic input data and the method is simulated for 2 users. Additive White Gaussian Noise (AWGN) channel is used. The individual power for 2 users has been calculated with equation (3) using GPA scheme and compared with other schemes to validate the proposed formula. The implementation system is designed to observe the BER performance of our 
proposed GPA scheme in noisy environment and also to compare our scheme with conventional other schemes.

\subsection{Calculated Power Comparion of GPA Scheme with Other Schemes for NOMA}

We have considered total power $P$ to be 100 watt for all users. In the table, the number of user is 2 and their power is calculated by using different types of power allocation technique. We have considered $20 \%$ of total power for User 1 and $80 \%$ of total power for User2 for Arbitrary Power Allocation scheme [9]. We have calculated the power of User 1 is $\alpha_{1} * P$ watt and User $2 \alpha_{2} * P$ by using the FTPA scheme [10]. Power allocation factor $\alpha_{1}$ for User1 is 0.17 and $\alpha_{2}$ for User2 is 0.82 and they are calculated by using fairness index 0.7 as of [11]. Finally we have calculated the power by using our proposed GPA scheme. The calculated power for 2 different users with different power allocation schemes have been shown in Table 2 and Fig.4.

TABLE2: CALCULATED POWER FOR 2 USERS USING DIFFERENT SCHEMES

\begin{tabular}{|c|c|c|c|}
\hline \multirow{2}{*}{$\begin{array}{c}\text { Power } \\
\text { allocation } \\
\text { schemes }\end{array}$} & \multicolumn{2}{|c|}{ Power in Watt } & \multirow[b]{2}{*}{$\begin{array}{c}\left(\mathbf{P}_{1}+\mathbf{P}_{2}\right) \approx \\
\mathrm{P}\end{array}$} \\
\hline & $\begin{array}{l}\text { Power of } \\
\text { User 1, } P_{1}\end{array}$ & $\begin{array}{c}\text { Power of } \\
\text { User 2, } \\
P_{2}\end{array}$ & \\
\hline $\begin{array}{l}\text { Arbitrary } \\
\text { Power } \\
\text { Allocation }\end{array}$ & 20 & 80 & 100 \\
\hline FTPA & 17 & 82 & 99 \\
\hline GPA & 18 & 81 & 99 \\
\hline
\end{tabular}

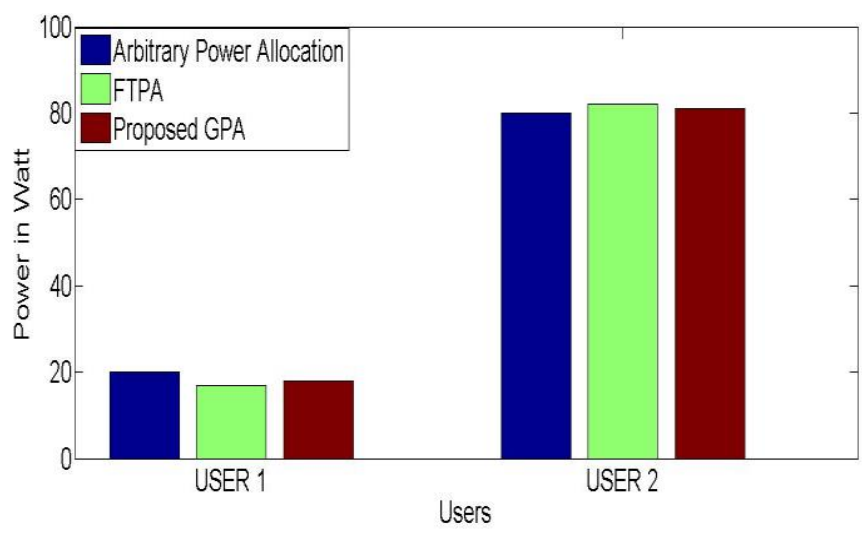

Fig. 4.Calculated powers with different power allocation schemes of NOMA for 2 users

The Table 2 and Fig. 4 show that the calculated power for both the users is almost similar for different schemes. Therefore, we can say that our proposed scheme shows the similar result with other schemes but our proposed GPA scheme is simple and there is no need to optimize any arbitrary values for efficient calculation of power for different users.

\subsection{Performance Comparion of GPA Scheme with Other Schemes for NOMA}

The BER performance of proposed GPA scheme and two other conventional power allocation schemes are simulated under implementation of convolutional channel coding techniques with 
BPSK digital modulation technique. The results are presented and compared in Fig.5. It is observed from the figure that GPA scheme shows a satisfactory BER performance and the performance is similar with two other conventional power allocation schemes in the context of BER.

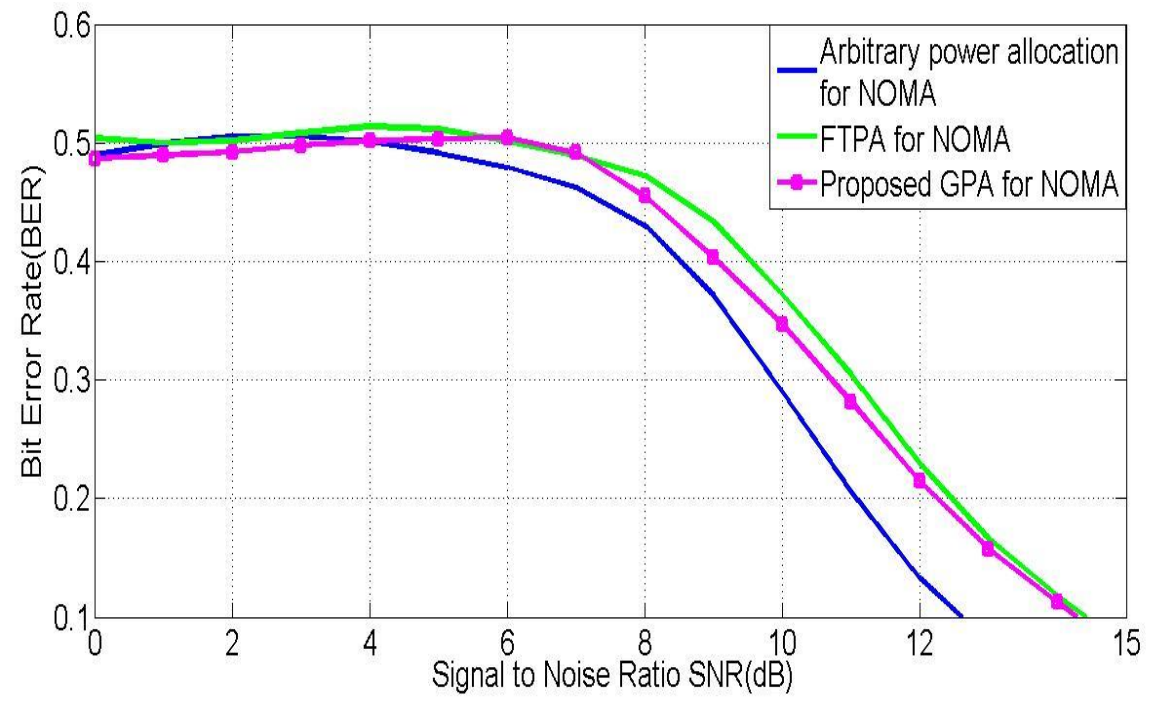

Fig. 5.Performance comparison of proposed GPA scheme with other existing schemes using BPSK modulation technique.

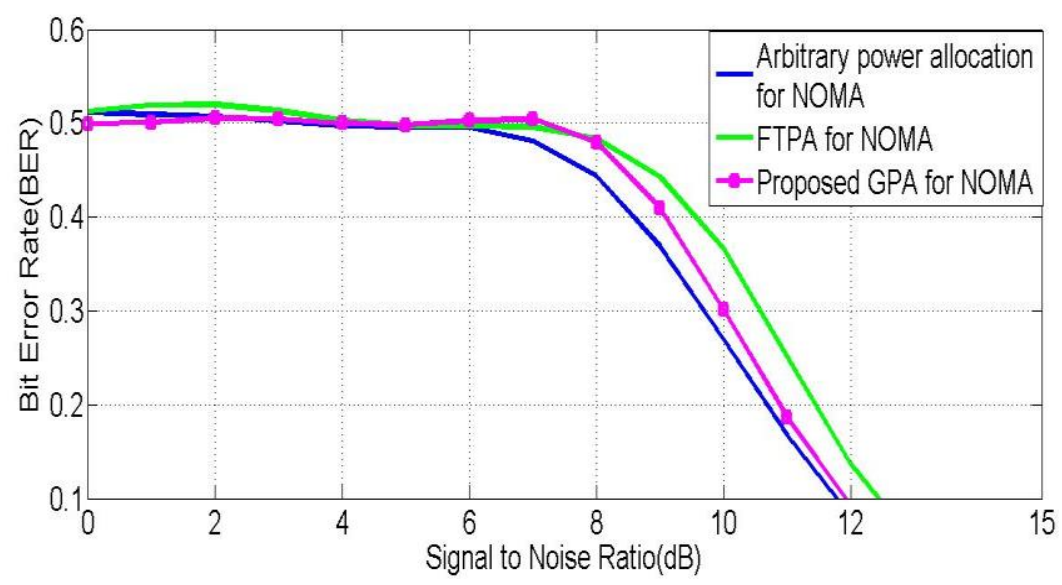

Fig. 6.Performance comparison of Proposed GPA scheme with other existing schemes using 4-QAM modulation technique.

Fig.6 shows the BER performance with different power allocation schemes for NOMA using 4QAM modulation technique. In this figure, we observe that the GPA scheme shows similar BER performance in case of 4-QAM digital modulation technique also. Therefore the proposed GPA scheme shows consistent BER performance for different modulation techniques as well.

\section{CONClusion And Future Works}

Power allocation need to be effectively addressed for NOMA. In this paper, we have proposed a Generalized Power Allocation (GPA) scheme which is simple but effective. GPA scheme does not need to use any arbitrary value for power calculation of different users and the calculated 
powers are distinguishably different for different users. We demonstrated by calculation and simulation that GPA scheme satisfies the conditions and prerequisites of basic NOMA design. The calculated total power using GPA scheme in NOMA based wireless communication system shows only $01 \%$ variation than the arbitrary power calculation for multiple users. The simulation results show that GPA scheme performs in a similar way to other conventional power allocation schemes for NOMA. Therefore this simple GPA scheme is targeted to address the challenge of power allocation in NOMA and can enhance its performance for future wireless communication. Though the BER performance of GPA scheme is compatible with other conventional power allocation schemes for NOMA, the overall BER performance is needed to be improved for future wireless communication system. Therefore, our future target is to accomplish an effective BER minimization technique for NOMA based wireless communication system.

\section{ACKNOWLEDGEMENTS}

This work has been done under the scholarship support of NST fellowship program of the Ministry of Science and Technology, People's Republic of Bangladesh and the work has been conducted in the Communication Signal Processing Laboratory of the Department of Information and Communication Engineering, University of Rajshahi, Bangladesh.

\section{REFERENCES}

[1] A. Zanella, N. Bui, A. Castellani, L. Vangelista, and M. Zorzi, "Internet of Things for Smart Cities", IEEE Internet of Things Journal, vol. 1, pp. 22-32, Feb 2014.

[2] H. Zhu, "Radio Resource Allocation for OFDMA Systems in High Speed Environments", IEEE Journal on Selected Areas in Communications, vol. 30, pp. 748-759, May 2012.

[3] Y. Endo, Y. Kishiyama, and K. Higuchi, "Uplink non-orthogonal access with MMSESIC in the presence of inter-cell interference", 2012 International Symposium on Wireless Communication Systems (ISWCS), pp. 261-265, Aug 2012.

[4] T. Ahmed, "Comparative Study of Orthogonal Frequency Division Multiple Access (OFDMA) and Non-orthogonal Multiple Access (NOMA) for Wireless Communication", Project paper, Dept of ICE, University of Rajshai, 2015.

[5] C. L. Wang, J. Y. Chen, and Y. J. Chen, "Power allocation for a downlink non-orthogonal multiple access system", IEEE Wireless Commun. Lett., vol. 5, no. 5, pp. 532-535, Oct. 2016.

[6] Zhiguo Ding, Yuanwei Liu, Jinho Choi, Qi Sun, Maged Elkashlan, Chih-Lin I and H. Vincent Poor, "Application of Non-orthogonal Multiple Access in LTE and 5G Networks", IEEE Communications Magazine, Vol. 55, pp. 185 - 191, 2017.

[7] Y. Liu, Z. Qin, M. Elkashlan, Z. Ding, A. Nallanathan and L. Hanzo, "Nonorthogonal multiple access for 5G and beyond" Proc. IEEE, vol. 105, no. 12, pp. 2347-2381, Dec. 2017.

[8] Y. Cai, Z. Qin, F. Cui, G. Y. Li and J. A. McCann, "Modulation and multiple access for 5G networks," IEEE Commun. Surveys Tut., vol. 20, no. 1, pp. 629-646, First quarter 2018.

[9] Yuya Saito, Yoshihisa Kishiyama, Anass Benjebbour, Takehiro Nakamura, Anxin Li, and Kenichi Higuchi, " Non-Orthogonal Multiple Access (NOMA) for Cellular Future Radio Access", China Communications Volume. 12, Issue. Supplement, December 2015.

[10] Talgat Manglayev, Refik Caglar Kizilirmak, Yau Hee Kho, "Optimum Power Allocation for Nonorthogonal Multiple Access (NOMA)", IEEE 10th International Conference on Application of Information and Communication Technologies (AICT), 2016. 
[11]R.K. Jain, D.W. Chiu, and W.R. Hawe, "A Quantitative Measure of Fairness and Discrimination for Resource Allocation in Shared Computer System”, , pp. 20-21, Hudson, 1984.

[12] Shimei Liu, Chao Zhang and GangmingLyu, selection and power schedule for downlink non orthogonal multiple access (NOMA) system”, 2015 IEEE International Conference publications on Communication Workshop (ICCW), pp. 2561 -2565, 2015.

[13] S. Vanka, S. Srinivasa, Z. Gong, P. Vizi, K. Stamatiou, and M. Haenggi, "Superposition Coding Strategies: Design and Experimental Evaluation", IEEE Transaction on Wireless Communications, vol. 11, pp. 2628-2639, 2012.

[14] T. Ahmed, "Generalized Power Allocation Technique for Multiple Users with Non-Orthogonal Multiple Access (NOMA) in Wireless Communication System”, MSc Thesis, Dept of ICE, University of Rajshai, 2016.

[15]K. Higuchi and A. Benjebbour, "Non-orthogonal multiple access (NOMA) with successive interference cancellation for future radio access," IEICE Transactions on Communications, vol. E98.B, no. 3, pp. 403-414, 2015.

\section{AUTHORS}

Tofail Ahmed is a lecturer in the Department of Information and Communication Engineering, Bangladesh Army University of Engineering and Technology (BAUET), Natore, Bangladesh. He earned his B.Sc.(Engg.) degree and M.Sc. (Engg.) degree from University of Rajshahi, Bangladesh in 2015 and 2016 respectively. His research interest is on wireless communication.

Rubaiyat Yasmin is serving as a Professor in the Department of Information and Communication Engineering, University of Rajshahi, Bangladesh. She earned her $\mathrm{PhD}$ degree from Saitama University, Japan in 2011. She did her M.Sc. from Waseda University, Japan in 2005. She published a number of journal and conference papers in her area. Her research area is Communication Signal Processing.

Halida Homyara is serving as an Associate Professor in the Department of Information and Communication Engineering, University of Rajshahi, Bangladesh. She earned her PhD degree from Chubu University, Japan in 2004. She did her M.Sc. from University of Rajshahi, Bangladesh. She published a number of journal and conference papers in her area.

M.A.F.M. Rashidul Hasan is serving as a Professor in the Department of Information and Communication Engineering, University of Rajshahi, Bangladesh. He earned his D.Engg. degree from Saitama University, Japan in 2012. He was a visiting researcher in Waseda University, Japan from 2004 to 2005. He published a number of journal and conference papers. His research area is Signal Processing.
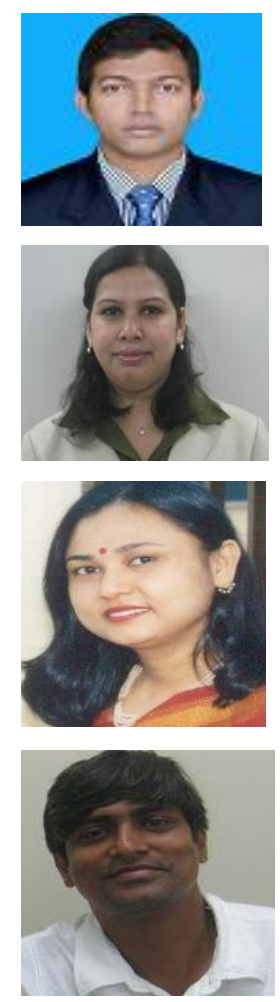